\title{
NOTAS SOBRE LA AMBICIÓN FEMENINA COMO ORIGEN DE LA AMBIGÜEDAD ACTANCIAL EN ANNE MARIE DE LUCIEN BODARD
}

ANA SOLER PÉREZ

Universidad de Zaragoza

Lucien Bodard, periodista de profesión, inicia tardíamente ${ }^{1}$ su carrera como escritor en los años setenta. En su obra literaria subyace de manera obsesiva un doble universo. Por un lado, aparece el macromosmos del mundo chino, ámbito contradictorio y fascinante, lugar de encuentro de ignominía, belleza y monstruosidad y donde el espectáculo de la muerte omnipresente se codea íntimamente con el de la vida. En esta unidad macrocósmica se enmarca el espacio anímico y familiar del narrador, en el que destaca la figura de una mujer Anne Marie que da precisamente título a la novela ${ }^{2}$ que en 1981 fue galardonada con el Premio Goncourt ${ }^{3}$.

1 Escribe su primera novela Monsieur le Consul a los sesenta años.

2 Se inserta en una trilogía constituida por Monsieur le Consul (1973), Le fils du Consul (1975) y Anne Marie (1981), publicada en la editorial Grasset-Fasquelle. En este sentido, Bodard entronca con la tradición literaria de las sagas familias la China de principio de los años veinte mienzos de nuestra centuria. Monsieur le Consul describes commerces médiévaux et les trafics du víctima de una profunda dicotomía «où s'amalgains, de machines, de dollars et la Chine de la faXXème siècle. La Chine des étrangers fous de gai Max OLIVIER-LACAMP, «La Chine des années fomine, des guerres civiles et des mast

Este fresco será retomado posteriormente en Le fils du Consul que insiste sobre la crueldad, atrocidad y decadencia vividas desde su nacimiento en la provincia de Sseu-Tch'ouan por el pequeño Lulu.

Grégory Pons establece un paralelismo que alude precisamente a la edad avanzada del au3 Grégory Pons establece un paralelismo que alude precisarnente a la République, la "Répu-
tor: «Après la France politique, qui a élu François Mittérand président de la
blique des Lettres" vient d'honorer un grand-père: Lucien Bodard, 67 ans». Cf. «Un Goncourt sans imagination» in Le Spectacle du monde/Réalités, 237, dic. 1981, pp. 94-96, p. 94 
Esta obra constituye, ante todo, una historia sobre el comportamiento femenino. Su núcleo temático gira en torno a la ambición y al afán de poder femeninos y sus formas de actualización, encarnados sobre todo en la persona de Anne Marie Bonnard, madre del narrador, Lucien. El juego Bonnard/Bodard ${ }^{4}$ no es sino la marca de rechazo de un pacto autobiográfico explícito manifestado por el novelista. Monsieur le Consul ${ }^{5}$ inauguró esta transformación, que se vio truncada en la siguiente obra de Bodard Le fils du Consul ${ }^{6}$. El contrato de lectura de Anne Marie, en términos de Lejeune, nos induce a leerlo como una obra novelesca, de la que se desprende sin embargo un profundo carácter autobiográfico ${ }^{7}$.

Cinco ejes vertebran la novela y articulan la red urdida por la protagonista, para lograr su proceso volitivo de mejora. Así, podemos destacar las relaciones de Anne Marie 1) con Albert, su marido y 2) con Lulu, su hijo. Ambos integran a su vez un primer grupo, constituido por personas pertenecientes al círculo familiar de Anne Marie, que denominaremos grupo endogámico. Entre el elenco de personas que se adscriben al ámbito de lo no familiar, se hallan 3) André 4) su mujer Edmée y, por otro lado 5) las hermanas Rose y Diane, todos ellos integrantes del grupo exogámico.

4 El autor es Lucien Bodard y el narrador Lucien Bonnard. Esta total coincidencia en el nombre y casi coincidencia en el apellido alude a uno de los casos de indeterminación contemplados por Philippe Lejeune. En su tablero de doble entrada donde aparece por un lado la alternativa novelesco/autobiográfico para el pacto y por otro la de diferente/semejante para el nombre del personaje con relación al del autor, la situación presente no responde formalemente a las manifestadas en esta tipología. Philippe Lejeune, Moi aussi, Paris, Ed. du Seuil, 1986, pp. 24-25. Se trata de un nombre sustituido que se halla en un estadio intermedio entre un nombre real y uno imaginario. Por ello, es percibido como inventado y se supone que designa a una persona real, en este caso el autor.

5 El propio autor reconoce su naturaleza autobiografica, que sin embargo rehuye formular explícitamente. Véase a este respecto el artículo de Jean-Louis EzINE, «Lucien Bodard entre le désanchantement et la passion* in Les Nouvelles Litteraires, $2401,1{ }^{\text {ex }}$ oct. 1973, p. 3.

6 Le fils du Consul introduce un cambio sustancial en el seno de la trilogia, ya que aunque trata de la misma temática, transcurre en idéntico período y comparte el mismo narrador, aparece como kune véritable autobiographie qui n'a rien perdu de ses qualités romanesques. Le consul est redevenu, comme dans la réalite, Albert Bodard (et non plus Bonnard)». Pierre de BolsDefFre, «Lucien Bodard: Le fils du Consul $\star$ in Revue des deux Mondes, oct. 1975, pp. 649-653, p. 651.

${ }^{7}$ Los datos que aparecen en esta obra cobran autenticidad ya que también lo hacen en Le fils du Consul que sí es una verdadera autobiografía. También el reconocimiento de numerosas similitudes tanto acerca del protagonista y su familia como de personas políticas y hechos históricos apoyan esta afirmación. Así, los Masselot, anfitriones de Anne Marie y su hijo designan en realidad al matrimonio formado por Philippe Berthelot y su esposa. Este fue director de asuntos políticos del Quai d'Orsay de 1913 a 1920. Cf. Pierre de BolsDefFre, «Lucien Bodard: Anne Marie » in Revue des deux Mondes, oct.-dic. 1981, pp. 149-160, p. 157. 
El eje semántico del Ser frente al Parecer recorre integramente la novela, poniendo de manifiesto el doble papel actancial que representan sus personajes.

\begin{tabular}{|c|c|c|}
\hline & Ser & Parecer \\
\hline Anne Marie & $\begin{array}{l}\text { agente voluntario de un proceso } \\
\text { de mejora }\end{array}$ & $\begin{array}{l}\text { paciente victima de un estado } \\
\text { insatisfactorio (situación de } \\
\text { sacrificio }^{8} \text { ) }\end{array}$ \\
\hline Albert & $\begin{array}{l}\text { víctima de un proceso } \\
\text { de degradación }\end{array}$ & agente voluntario de prestación \\
\hline Lulu & $\begin{array}{l}\text { víctima de un proceso } \\
\text { de degradación }\end{array}$ & beneficiario de mejora \\
\hline André & paciente víctima de un engaño & agente voluntario de prestacion \\
\hline $\begin{array}{l}\text { Edmée } \\
\text { (Rose y Diane) }\end{array}$ & $\begin{array}{l}\text { agente }{ }^{9} \text { voluntario de obstrucción } \\
\text { en potencia }\end{array}$ & agente voluntario de prestación \\
\hline
\end{tabular}

Con ello se consigue recrear el ambiente de hipocresía reinante en una clase concreta de la sociedad francesa de los años veinte: la burguesía.

La trama de la novela se basa en el quebrantamiento de una situación inicial perfectamente equilibrada, a causa de la llegada de una persona intrusa: Anne Marie. Ésta intenta integrarse en un círculo social hermético y elitista presidido por el director de Asuntos Exteriores André Masselot y su esposa Edmée. La tarea difícil de la heroína como agente voluntario consiste en ser admitida en dicho cenáculo como un miembro de pleno derecho, recurriendo a toda clase de artimañas para lograr su propósito. Consigue doblegar a las personas de su entorno convirtiéndolas en víctimas de degradación o de engaño, resul-

8 Frente al supuesto beneficiario de un proceso de mejora: Lulu.

9 En realidad, Edmée es un agente virtual. Según Bremond tres causas pueden motivar que un rol de paciente no se transforme en agente. Basta imaginarse una persona que permanece sin reacción frente a unos sucesos que le afectan: cuando no es consciente de ello, cuando no ve ningún motivo para replicar o cuando una plena impotencia se lo impide. Claude BREMOND, Logique du récit, Paris, Éd. du Seuil, 1973, p. 174. En este caso no es que Edmée no vaya a intervenir, s6́lo se introduce un compás de espera para que se produzca dicha reacción. 
tando ser paradójicamente el único agente voluntario, a pesar de su aparente situación de indefensión. Su treta conlleva implícitas dos acciones: una «dissimulation» y una «simulation». La señora Bonnard disimula lo que es, simula lo que no es y sustituye lo que no es por un «parecer», que el engañado acepta como «un ser verdadero». Para ello dispone de una serie de ayudantes y oponentes.

Dentro de la categoría de los ayudantes podemos destacar en un principio a su marido, Albert. La aceptación por su parte de una soledad sacrificada despeja el camino a su esposa. En este sentido, Lulu desempeña un papel muy similar. En una sociedad de moral puritana aunque a menudo hipócrita, representa un salvoconducto para su madre, quien justifica su alejamiento "forzoso" de su marido por el bien de su hijo. Así, su acción interesada cobra la apariencia de un sacrificio maternal, que exalta su valor y generosidad.

Resentida contra la vida, por haber nacido en el seno de una humilde familia de provincias, Anne Marie utilizó su matrimonio como escapatoria a un destino injusto. Abocada a vivir en la mediocridad el resto de su existencia por la situación económica de sus padres, optó por un matrimonio de conveniencia, sin amor, para alcanzar un estatus social de otra forma inaccesible. Lograda esta posición y convertida ya en la señora del cónsul en China su nueva existencia en tierras orientales le recuerda su provincianismo. Esta vez se escuda tras su papel de madre para abandonar ese país y alejarse de su propio marido. En ambos casos, su naturaleza femenina le brinda las vías para resarcirse de una situación inicial desfavorable. Su asentamiento provisional en París funciona como el primer paso de su estratagema. La capital francesa, ciudad de sueños e ilusiones, enclave del mundo de la política y de las finanzas, reino de la moda, del lujo y de la aristocracia conforma su meta inmediata. Su vinculación a la diplomacia se alza como trampolín para acceder a la esfera de la mundanidad parisina, donde sus dotes para manejar las relaciones personales conseguirán el resto.

Por ello, Albert, que ante los demás aparece como agente voluntario de prestación resulta ser en realidad una paciente víctima. En cuanto a Lulu, que aparece en un principio como paciente beneficiario de un proceso de mejora, se desvela como una paciente víctima de un proceso de degradación. La alegría por su inscripción en uno de los colegios de mayor prestigio de Francia y por la compañía de su madre, que pensaba disfrutar en solitario, se desvanece pronto. El régimen de internado impuesto por la decisión materna genera por vez primera una sensación hasta entonces desconocida para él, la soledad, a la vez que inicia una serie de vejaciones crueles por parte de sus nuevos compañeros. Estos son asimilados a símbolos que Durand califica como «thériomorphes»: «es- 
saims de flèches», «insectes, bestioles méchantes, rongeuses, mangeuses» (pp. 212-213).

La designación impersonal de su madre en el propio título señala un primer atisbo de la relación tan peculiar existente entre estos dos seres: se trata de un simple deíctico, frío y objetivo, sin expresión afectiva alguna. Para este niño de diez años, educado y mimado según las costumbres chinas por su ama $\mathrm{Li}$, su convivencia en solitario con su progenitora pone al descubierto una realidad hasta entonces desapercibida: Anne Marie resulta ser para él una verdadera extraña. Esta sensación se desprende en su primer contacto con Francia, tras el largo exilio chino. Frente a la actitud embriagada de su madre, que contempla eufórica los monumentos y las gentes de la capital, la visión del pequeño es antitética y disfórica. La ciudad le resulta muerta, los lugares, a pesar de su gentío, le parecen desiertos. Mientras Anne Marie se muestra exultante, Lulu se siente oprimido en medio de esta arquitectura agobiante. La tierra prometida, la patria querida le defrauda. La posesión simbólica de su madre en esta ciudad inhóspita choca con la cruda realidad: Lulu sólo constituye para ella un estorbo. Sus palabras de rechazo hacia ella, al descubrir sus verdaderos planes, no pueden ser más explícitas "C'est sur ma mère que je pisse» ${ }^{10}$ afirma en un momento dado. Esta doble decepción alude según Pierre de Boisdeffre, «à la mère naturelle et à la mère spirituelle». «Sa mère spirituelle, cette France qui, vue de Chine, lui paraissait si grande, si généreuse, si imposante, n'était qu'un trompel'oeil»"1.

André Masselot también caerá en las redes de Anne Marie y se convertirá en su principal ayudante dentro del círculo. Como persona del sexo masculino, no ha sido insensible a los encantos de esta mujer fatal que con su atractivo y naturalidad cuidadosamente estudiados, emprende con él un juego de seducción tan sutil que no es percibido por éste, aunque sí por su mujer. Contrariamente a los demás, sólo actuará como ayudante no poniendo trabas al proyecto de su huésped. Este amparo masculino es consecuencia de la «actualización de medios» dispuestos por Anne Marie mucho antes de su desembarco en París. André no se percata del anzuelo, a diferencia de su esposa, que decide voluntariamente entrar en el juego de su rival para preparar una contraofensiva posterior.

Edmée, Rose y Diane pertenecen al ámbito femenino por ello Anne Marie, a pesar de su intento, no conseguirá engañarles. Sin embargo, para salvar las apariencias, van a convertirse a su pesar en ayudantes de la protagonista. Entre

10 Cf. Lucien Bodard, Anne Marie, Paris, Grasset-Fasquelle, 1981, p. 301.

11 Pierre de BOISDEFFRE, «Lucien Bodard: Anne Marie», art. cit., p. 150. 
ellas, cabe destacar la primacía de Edmée frente a las dos hermanas, que son únicamente fieles acólitas de la primera. Se encargan de ponderar lo que manifiestan los Masselot, de ensalzar sus hechos y dichos, en definitiva perpetúan la armonía y las normas específicas del círculo social. Junto a sus anfitriones, encarnan los valores morales sempiternos de la vieja Francia y constituyen una de las bases sobre la que descansa esta sociedad.

Dentro del cenáculo, la señora Bonnard va a compensar su falta de cultura inicial con su ingenio innato y su amena conversación que van a convertirla pronto en invitada de excepción ${ }^{12}$. Su mérito consiste en lograr destacar personalmente a través de unas iniciativas aparentemente altruistas. Edmée no desconoce la astucia de Anne Marie, como tampoco ignora cuánto detesta su marido las disputas domésticas. Por ello decide ayudarla y no enfrentarse abiertamente con ella. Cumpliendo el viejo refrán chino, decide unirse a su enemiga para poder vencerla. Esta ayuda se manifiesta en momentos claves dentro de la trama, que forman pequeños peldaños que Anne Marie va ascendiendo paulatinamente, como la primera visita realizada a los Masselot y a las hermanas, auténtica prueba de fuego para la protagonista .

Entre los oponentes, el primero en actuar es su propio hijo, Lulu, quien, si bien en un primer momento es aliado de su madre, se vuelve contra ella tras verse abandonado. En las pocas cartas intercambiadas entre ellos contrasta el tono de súplica del niño con la indiferencia mostrada por la señora Bonnard. Además, los modos de reproducción elegidos para transcribir las misivas corroboran esta situación anímica de desamparo del pequeño ${ }^{13}$. Único contacto entre los tres miembros de la familia tras su separación, éstas juegan un papel fundamental en la trama: suponen el medio de obstrucción al proyecto de Anne Marie por parte de Lulu primero y de su marido posteriormente.

En efecto, Lulu, consciente del papel de víctima que comparte con Albert, decide vengarse de su progenitora y adopta la función de «influenciador» de su

12 La posesión de la palabra en el seno del círculo señala la jerarquía de los invitados, a la vez que mide la estima que éstos despiertan en sus anfitriones. Anne Marie se adueña de ella y la monopoliza merced a sus dotes de conversadora, que le permiten cautivar a las personas de su alrededor. Sólo André brilla más que ella, lo que instaura una «conversation entre André, (...) et Anne Marie qui répond. Duo.» Cf. Lucien Bodard, Anne Marie, op. cit., p. 126.

${ }^{13}$ Las cartas de Anne Marie simbolizan el restañamiento del cordón umbilical roto. La primera, esperada con una ansiedad febril, aparece traspuesta en estilo directo, lo que subraya su importancia. Ocurre lo mismo con la primera carta paterna, aunque sea recibida con menos entusiasmo. Sin embargo, las siguientes, puras repeticiones de las anteriores son reflejadas en estilo indirecto libre por el narrador. El contenido de éstas se centra en puras recomendaciones domésticas y desde luego está exento de muestras de afecto. Las posteriores cartas se muestran todavía más resumidas y aparecen en estilo narrado. 
padre. Como informador, comunica a Albert el proceso que está llevando a cabo su madre y le infunde sospechas sobre una relación más allá de la pura amistad entre André y ella. En este momento, y como consecuencia de ello, existe un cambio de dramatis personae en el seno de los personajes: Lulu y Albert dejan de ser pacientes para pasar a ser agentes obstructores. Pero en el caso del marido, esta alteración de funciones resulta momentánea. Las amenazas de Albert se quedan en meras palabras y pronto la señora Bonnard recupera su fuerza por medio de una seducción, en el sentido bremondiano del término ${ }^{14}$ : la promesa de una próxima paternidad. Ello frustra la obstrucción de Albert, que recobra su función de paciente aliado a la espera de una compensación futura y se comporta como acreedor de la beneficiaria de su ayuda. Tanto Anne Marie como Lulu saben que a través de esta treta, la primera ha salvado el obstáculo y que su plan sigue inexorablemente su curso.

El momento en que Anne Marie pide consejo a los Masselot sobre la posibilidad de establecerse definitivamente en París constituye el clímax de la novela. El rol de influenciador-embaucador de Anne Marie cobra toda su fuerza en la medida en que va a convencer a sus anfitriones para que aprueben su proyecto. La respuesta de éstos sólo ocupa unos segundos en el tiempo de la historia, que sin embargo son alargados en el tiempo del relato por medio de una pausa narrativa. La perspectiva del narrador orienta la descripción tanto de los pensamientos como de las palabras y actitudes de las dos rivales y la información desplegada se desarrolla en dos planos. Por una parte, existe la información explícita formulada por estas damas en el transcurso de su conversación y por otra el contenido implícito de sus verdaderos pensamientos. El sentido de las palabras aduladoras y lisonjeras emitidas por las dos mujeres se opone al que esconden sus auténticas intenciones: Anne Marie pretende deshacerse de su marido y Edmée salvaguardar su matrimonio, alejando a su rival. El contraste entre el extenso debate interno de la anfitriona, que se prolonga a lo largo de tres páginas y las réplicas breves y rápidas del diálogo emitido da buena cuenta de este proceso de oposición ser $v s$ parecer.

Elle s'interrroge. Satisfaire Anne Marie, c'est la garder en France, et, une fois installée ici, Edmée en est certaine, Anne Marie (...) s'incrustera et, tôt ou tard, ce sera sa ruine (p. 659). Edmée sait que le ton d'André

14 Es decir, en dicotomía con el término de intimidación que es la otra modalidad de negociación. La seducción tiende a inspirar el deseo de un servicio que se quiere ofrecer a cambio mientras que la intimidación quiere inspirar el temor a un posible prejuicio. $C f$. Claude BrEmond, op. cit, p. 264. 
décidera de son comportement à elle, en attendant, elle n'est qu'enchantement. «Chère Anne Marie, que me dites-vous là» Ce sera merveilleux. Comme cela, nous vous aurons souvent auprès de nous (p. 662).

La respuesta afirmativa de los Masselot que cierra el relato supone la tercera fase del proceso secuencial (virtualidad, actualización y resultado). Anne Marie ha logrado embaucar a sus anfitriones lo que conllevará en el futuro inmediato la persuasión de un tercero: Albert. Éste se verá forzado a asumir el consejo dado por su amigo y superior lo que implica el mantenimiento de su estado de degradación a la vez que abre la posibilidad de un proceso degradador para Edmée. Está claro que Anne Marie vence en todos los frentes. Su proceso de mejora pone de manifiesto el futuro desarrollo de una semilla de discordia que la novela ha ido sembrando entre sus líneas. Por ello, la novela predice para el futuro una lucha a muerte entre las dos rivales que, según palabras del narrador, acabará con el repudio de una de ellas.

Así, en esta búsqueda de un espacio social y personal ${ }^{15}$, Anne Marie tiene en la persona de Edmée, a su oponente más temible ${ }^{16}$. Ello se debe a la similitud de rasgos que comparten ambas mujeres, unas advenedizas, salidas de la nada, que han logrado labrarse una posición social a priori inaccesible gracias a sus encantos seductores. En el ámbito masculino, es donde la protagonista consigue su triunfo más aplastante, al alcanzar el apoyo unánime tanto de Lulu, como de Albert y André, a pesar del intento de obstrucción de los primeros. El hecho de que su verdadero proyecto no haya sido descubierto por André, hombre dotado de una inteligencia superior a la común y acostumbrado a hacer y deshacer estrategias, a urdir y detectar planes, realza todavía más el mérito de Anne Marie en la consecución de su empresa.

\section{***}

Paradojicamente, y a pesar del cargo político de sus maridos, las mujeres aparecen, en esta novela, como las auténticas protagonistas. Ellas son las que toman decisiones, las que urden planes, las que en definitiva monopolizan los ejes del poder y del querer. Los hombres ven reducido su papel a meros espectadores pasivos. Su vulnerabilidad frente a las artes de seducción de las muje-

15 Éste conforma un tema literario tradicional dentro de la narrativa de ascension social y buenos ejemplos de ello son la picaresca o la novela en primera persona del siglo XVIII.

16 Y por asimilación a Rose y Diane también, pues las hermanas actúan en todo momento como una réplica de su anfitriona. 
res es manifiesta; caen fácilmente en las redes tejidas por éstas, que recrean la imagen de Eva, la mujer fatal. Las mujeres representan los agentes, ellos sólo actúan como pacientes.

Anne Marie ha conseguido alcanzar su proceso de mejora obteniendo prestaciones concedidas bajo engaño. La perfecta neutralización de las obstrucciones cruzadas por sus oponentes demuestra la sangre fría y la astucia de esta mujer que presenta una doble personalidad. Tras la apariencia de una madre atenta, se vislumbra en realidad la imagen de la madre terrible; tras la de esposa abnegada, la de una cónyuge infiel y, tras la amiga frágil y agradecida, la rival más temible.

El planteamiento de la trama como la realización de un proceso de mejora a través de una serie de peripecias no constituye, como se ha dicho, un tema novedoso. El proceso de integración en el medio parisino de Anne Marie Bonnard muestra los mecanismos por los que el personaje novelesco accede desde un medio alejado (ya sea una provincia en la literatura de los siglos anteriores, o el extranjero en el caso que nos ocupa y en otros de esta misma centuria ${ }^{17}$ ) a la metrópoli, símbolo de su pretensión de mejora individual y social. La simulación, la seducción, la ambigüedad entre ser y parecer, la manipulación de su propia familia, demuestran la ambición sin límites que alimenta y justifica a Anne Marie. Estos elementos tampoco son ajenos al tema de la ascensión social. Sin embargo, existe una diferencia notoria: la mejora era protagonizada mayoritariamente por un ser humano de sexo masculino. La novedad es que Bodard repite, en Anne Marie, la estructura pero no con un protagonista sino con una protagonista. Es la mujer quien asciende en este caso en la sociedad, quien busca su lugar y su triunfo, quien se sirve de «armas de mujer» y quien impone su determinación en un mundo todavía «dominado» por el hombre.

contemporáneos consagrados ilustran este lienómeno: La Goutte d'or de Michel Teumier et Désert de J. M. G. Le Clézio. En ambos casos se trata de unos africanos que viajan a París buscando una salida a su miseria, el héroe de Tournier es un muchacho llamado Idriss, mientras Le Clézio, en cambio, ha elegido a una joven, Lalla. 